\title{
Status of the Production of the CMS Silicon Tracker
}

\author{
Alexander Dierlamm on behalf of the CMS Tracker Collaboration.
}

\begin{abstract}
The CMS experiment at the LHC, CERN, will operate the largest tracker in the world entirely made of silicon detectors. The collaboration is facing the quality assurance work to prove the reliability and performance of the various parts produced by industry. A status report on the CMS Tracker is given, including the validation of single modules up to larger integrated substructures.
\end{abstract}

Index Terms-Compact Muon Solenoid (CMS), LHC, microstrip, performance, pixel, silicon, tracker.

\section{INTRODUCTION}

$\mathbf{C}$ OMPACT Muon Solenoid (CMS) is one of the two general purpose experiments at the Large Hadron Collider at CERN [1], [2]. The apparatus is designed to detect new physics at the $\mathrm{TeV}$-scale (Higgs physics, Super-Symmetry, extra dimensions).

To reach this goal a robust tracking and vertex reconstruction is necessary in a strong magnetic field ( $4 \mathrm{~T}$ ) and in the presence of a high background of minimum bias events (up to 24 per bunch crossing at high luminosity). Since silicon sensors enable a fast read-out and stand the harsh LHC radiation environment (up to $F_{\text {eq }}=1.6 \cdot 10^{14} \mathrm{n}_{1 \mathrm{MeV}} / \mathrm{cm}^{2}$ ), a tracker, entirely based on silicon detectors, was chosen for the CMS experiment. It is composed of an inner Pixel Detector with an outer radius of $10.2 \mathrm{~cm}$ surrounded by a Strip Detector, extending up to a radius of $110 \mathrm{~cm}$.

The Strip Detector consists of 6052 thin $(320 \mu \mathrm{m})$ and 18192 thick $(500 \mu \mathrm{m})$ single-sided silicon microstrip sensors with a pitch going from $80 \mu \mathrm{m}$ to $205 \mu \mathrm{m}$ and a strip length ranging from $6.6 \mathrm{~cm}$ to $11.7 \mathrm{~cm}$. This results in a total active area of more than $200 \mathrm{~m}^{2}$ and nearly 10 million read-out channels. In the first integration steps 31 different geometries of modules are produced, each consisting of a carbon fiber frame, one or two sensors and a front-end hybrid containing the read-out electronics. These modules are mounted on larger frames with geometries depending on the location in the Tracker. In the Outer Barrel a ladder structure ("rod") was chosen, the End Caps have disk segments called "petals," and for the Inner Barrel complete halfcylinders (shells) are equipped with detectors and electronics. These subassemblies are put together to form the subdetectors of the Strip Tracker, which are the Tracker Outer Barrel (TOB), the Tracker End Caps (TEC), the Tracker Inner Barrel (TIB), and the Tracker Inner Disks (TID) as shown in Fig. 1.

The Pixel Detector will be placed inside the Strip Detector after the first LHC test run. The barrel of the Pixel Detector has three layers with radii of 4,7 , and $10 \mathrm{~cm}$, for a total of 48 million pixels $\left(100 \times 150 \mu \mathrm{m}^{2}\right)$ with $300 \mu \mathrm{m}$ thick silicon as the active

Manuscript received October 15, 2004.

The author is with CERN, CH-1211 Geneva 23, Switzerland.

Digital Object Identifier 10.1109/TNS.2005.862935 volume. In addition, there are two pairs of disks with additional 18 million pixels (Fig. 2).

Both the Strip and the Pixel Detectors are situated within the 4 T magnetic field of CMS. An active cooling system will keep them at a temperature of $-10^{\circ} \mathrm{C}$ for most of the time to reduce the leakage currents and the reverse annealing effects of the silicon sensors.

\section{Production of the Silicon Strip Modules}

The Tracker collaboration follows a distributed production chain including about 20 institutes, which are responsible for the different integration steps and the quality assurance thereafter. More than 24000 silicon sensors have to be inspected and assembled to about 15000 modules. High precision and reproducibility is achieved using automatic assembly robots in a clean room environment. The production is expected to be completed in October 2005 for TIB, in December 2005 for TOB and in March 2006 for TEC.

\section{A. Hybrids}

The front-end hybrid production had to face three severe problems in the past year. The quality control found broken electrical lines near the connector of the flat kapton cable. The production was stopped and a stiffener was introduced so that bending near the connector is avoided. Another stop of the production was caused by the occurrence of weak bond connections made by the supplier. This was cured by optimising the bond parameters. In April 2004, open vias were detected on some hybrids. One affected via is on the reference voltage line. An open there causes the read-out chip (APV25) to charge up slowly and a large drift of the pedestals can be observed on a time scale of more than $10 \mathrm{~min}$. Since not all hybrids can be tested for such a long time, the problem was identified at a late stage, when a significant number of potentially defective hybrids had been fabricated. The problem has been thoroughly investigated: The hybrid consists of two kapton circuits glued together. During laser drilling of the $100 \mu \mathrm{m}$ wide vias, the softer glue melts faster and a cavity might form, which can hardly be covered by the metallization. The solution, which has now been qualified, is to introduce a third kapton layer, which stabilises the via, and to increase the via diameter to 120 $\mu \mathrm{m}$. In total 3799 good hybrids have been produced so far (out of 15000 needed) and 2602 were rejected because of the via problem. It was already shown that the production is able to sustain a rate of 1450 hybrids per month.

\section{B. Sensors}

The delivery of the thin strip sensors by Hamamatsu is completed. The quality of the thick sensors, delivered by ST Micro- 


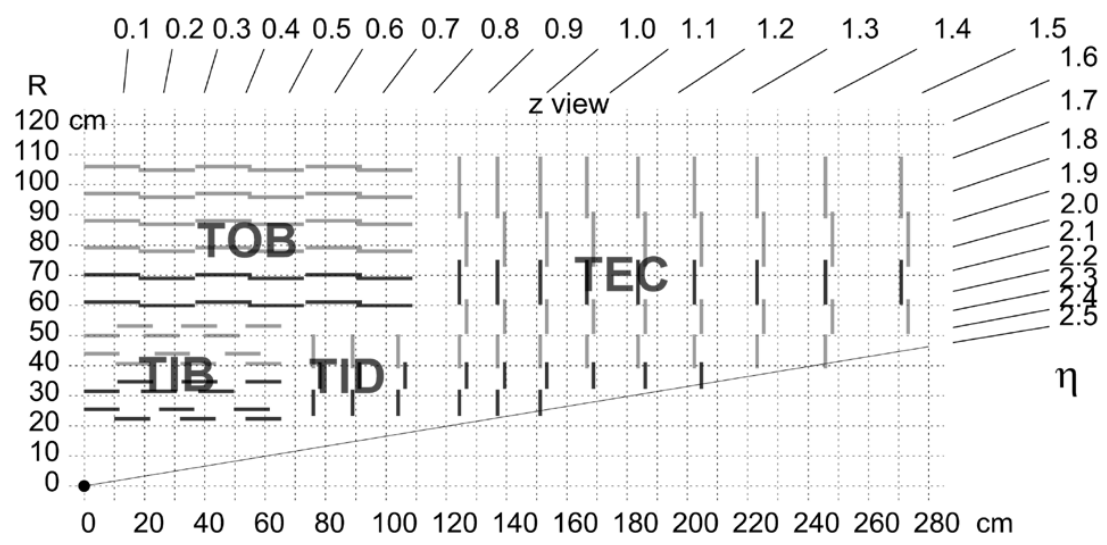

Fig. 1. One quarter cut of the CMS Silicon Strip Tracker. It has an outer diameter of $2.4 \mathrm{~m}$ and a length of $5.4 \mathrm{~m}$. Double-sided modules populate the regions with $20 \mathrm{~cm}<R<40 \mathrm{~cm}$ and $60 \mathrm{~cm}<R<75 \mathrm{~cm}$ (indicated with dark bars). The rest is equipped with single sided modules. The TIB has four layers of modules $(20-55 \mathrm{~cm})$, the TOB is made of six layers $(60-110 \mathrm{~cm})$, one TEC consists of nine disks segmented in seven to four rings and one TID has three disks.

electronics, has substantially improved during the qualification period but a degradation in terms of leakage current has been observed on some sensors, which might have its origin in a kind of microcorrosion at the guard/bias rings (Fig. 3). Distortions of the metallization edges affect the field line distribution and thus can increase the leakage current. The production at ST Microelectronics was stopped and Hamamatsu started producing thick sensors with excellent quality.

\section{Modules}

The module assembly centres have produced 2841 modules, of which 2345 are already bonded in the 14 bonding centres. During this first part of the production the yield was $94 \%$.

\section{INTEGRATION OF THE STRIP TRACKER}

With these modules the production of larger sub-assemblies has started for the four parts of the Tracker (Fig. 1). TEC-petals (Fig. 6) are equipped with up to 28 wedge-shaped modules and the rods of the TOB (Fig. 4) are populated with 6 single modules or double-sided pairs. These subassemblies are then mounted on larger support structures. Half-cylinders in the TIB are equipped with up to 54 modules, and the TID contributes six small disks with three rings. The procurement of the mechanical structures is advancing without major problems. In the following, the logistics of these integration steps are described in more detail.

\section{A. Outer Barrel}

The rod frames are produced in Helsinki, where they undergo a mechanical quality control. The precision pins, which define the positions of the module frames, are measured there. These carbon fiber frames are sent to CERN, where the electronics and the optical hybrids are mounted, and a detailed quality control is performed including electrical connection checks and functional tests of the components [3]. These cabling and testing procedures are now fully qualified and about 180 final rod frames of 720 have been produced since August 2004.

The rods are sent to the two module assembly centres at University of California, Santa Barbara, and FermiLab, Chicago.

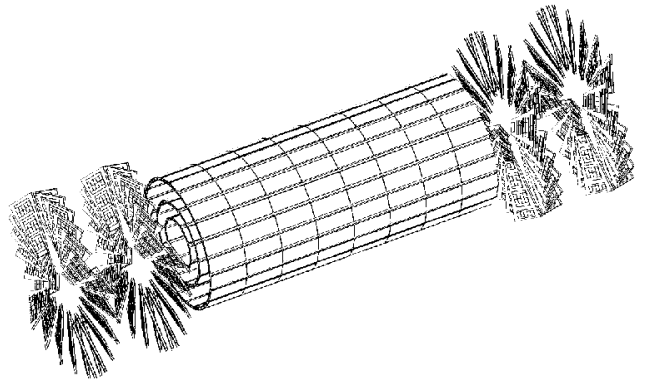

Fig. 2. Drawing of the CMS Pixel Detector. The barrels extend to $z= \pm 266.6$ $\mathrm{mm}$ with radii of about 4,7 , and $10 \mathrm{~cm}$. The disks are placed at $z= \pm 34.5 \mathrm{~cm}$ and $\pm 46.5 \mathrm{~cm}$ (a third pair of disks at $\pm 58.5 \mathrm{~cm}$ is planned).

After a first optical inspection, the modules are mounted on the frames and the rods are individually tested for their functionality. The rod qualification is completed by a 3-d burn-in test using up to eight rods, which are temperature cycled from room temperature down to the tracker operation temperature, and read out continuously. The assembled rods are then shipped back to CERN, where another setup is used to verify the functionality, and take signal data at room temperature using a beta source (Fig. 4). Until being inserted in the TOB, the rods are stored in an antistatic and ambient controlled environment. The assembly of the TOB wheel (Fig. 5) is in progress at CERN.

\section{B. End Caps}

Institutes at Aachen and Louvain produce the petal frames with an expected rate of one petal per day and per centre. Ten out of 432 frames are already produced. The precision machining of the module support inserts and the integration of the interconnect boards is done in Aachen for all petals. The control units, analog opto hybrids and modules are mounted in seven different petal integration centres, which are expected to produce one petal per week per centre. The petals undergo similar functionality tests as the rods [4]. The fully equipped petals are delivered to Aachen and Lyon, where the two End Caps are assembled. The carbon fiber structures (Fig. 7), service channels, inner shells and aluminum inserts are already available, and the assembly is to start soon. The complete End Caps are then delivered to CERN. 


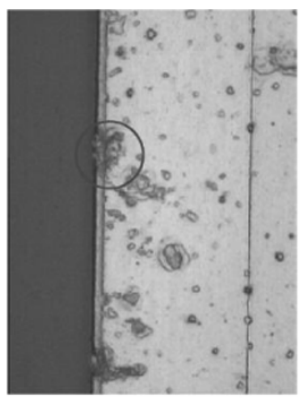

(a)

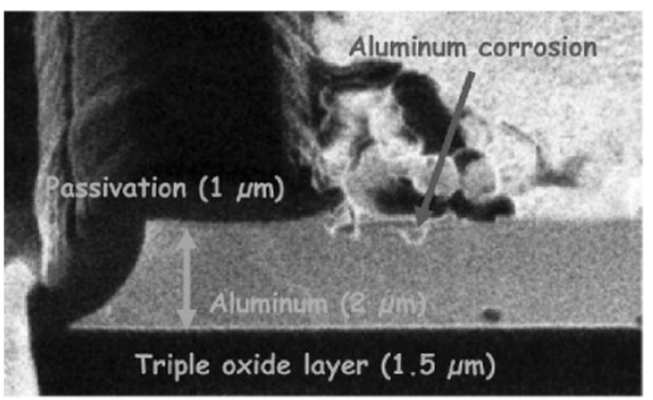

(b)

Fig. 3. The microscopic stains are caused by corrosion of the aluminum. Bad edges of the metallization might increase the field strength there causing a higher leakage current. (a) Microscopic view of dots and stains on the metallization. (b) Focused ion beam image of a cut through the stain on the left.

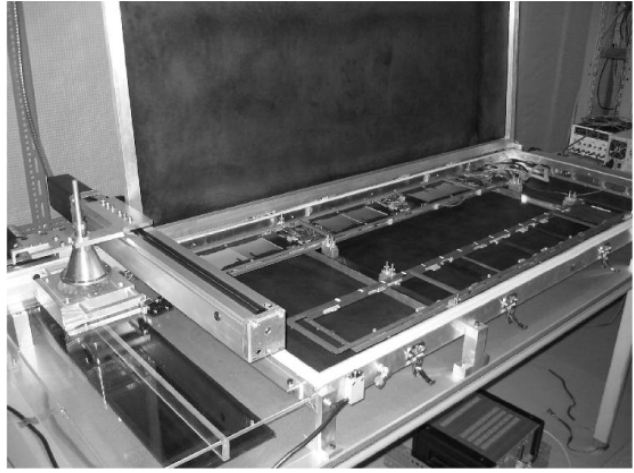

Fig. 4. Rods in the Rod Reception Test setup at CERN, which can hold up to three rods and scan them with a beta source to measure the signal-to-noise ratio.

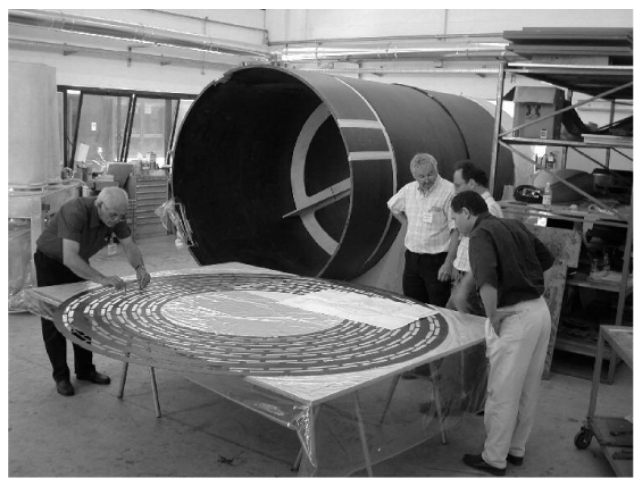

Fig. 5. Picture of the assembly of the TOB mechanics ('wheel').

\section{Inner Barrel and Disks}

The structure of the TIB (see Fig. 8) is different from the TOB and TEC, since there are no sub-assemblies, but the support structures are equipped directly with modules. There are 16 parts (forward and backward barrels with four layers of two half cylinders each), which are being equipped with cooling pipes first. Then the modules are integrated on the structure, and interconnected via a mother cable. A replica of a layer 3 half cylinder was equipped with modules in Pisa and operated in a beam test this year.

The production of the mechanics for the TID and the procurement of the mother cables have started. A TID pilot integration exercise is planned for March of 2005. The TID will be assembled after the completion of the TIB in Pisa and Torino.

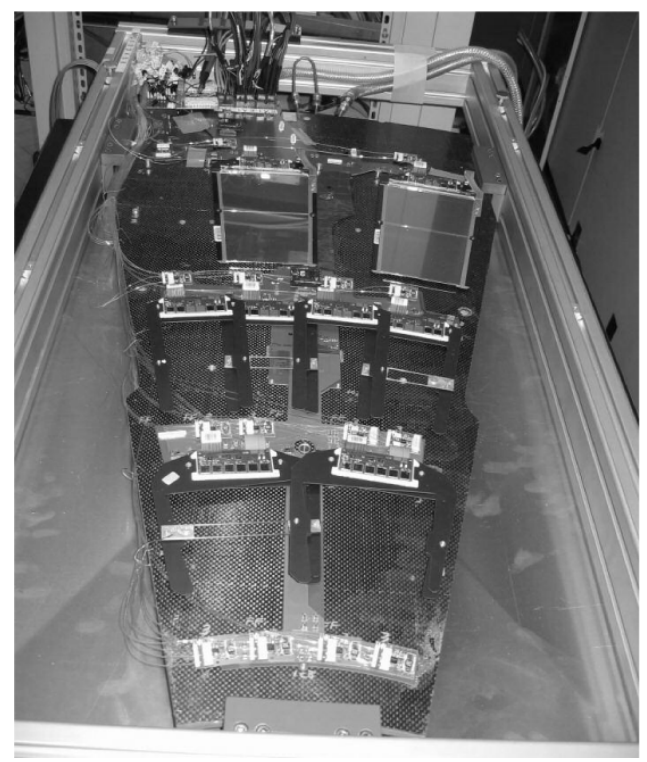

Fig. 6. Petal during integration and testing at Karlsruhe.

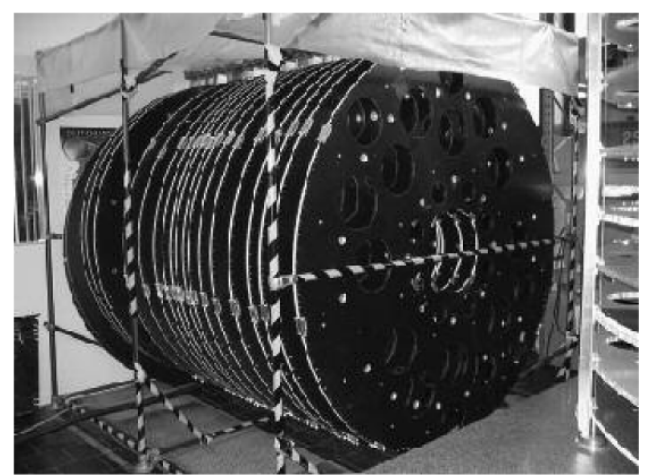

Fig. 7. Carbon-fiber structures for the TEC disks.

\section{Tracker Integration}

The delivery of the Tracker Support Tube (Fig. 9) was celebrated in September 2004. All subdetectors will be commissioned and mounted into this support tube at CERN. The commissioning of the subdetectors can profit from an elaborated DAQ system (see [5] for the Front End Driver and [6] for the Control system) and beam test experiences. 


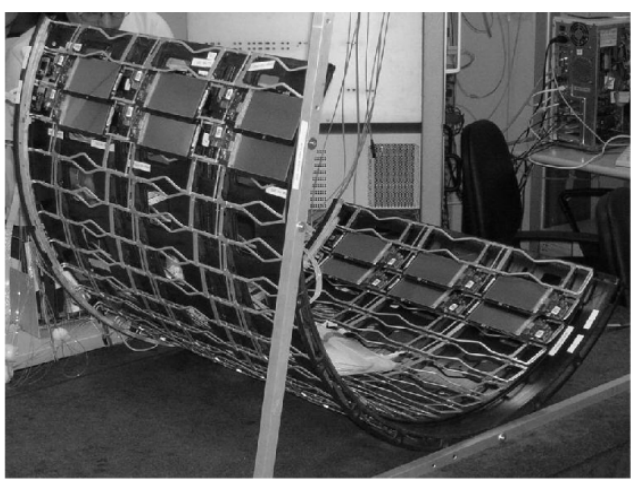

Fig. 8. TIB half-cylinder with cooling pipes glued and first detector strings installed.

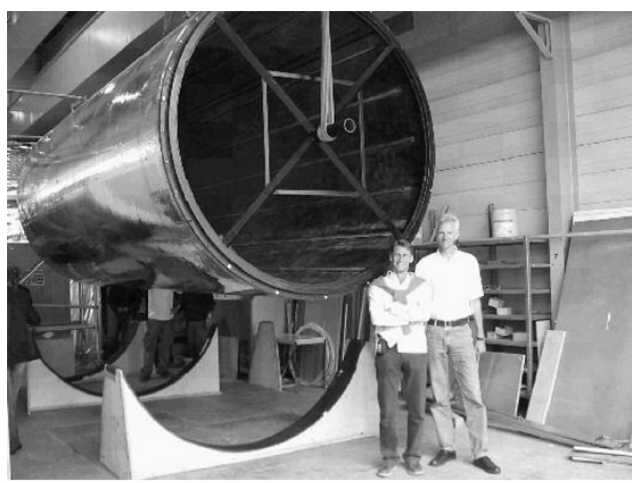

Fig. 9. The Tracker Support Tube has been delivered at CERN.

\section{BEAM TESTS}

The first subassemblies produced were also tested in two 25 ns-test beams at the SPS, CERN, which took place in May and October 2004. In May one front and one back petal from the TEC (at low temperatures) [4] and in October a half-shell of the TIB (at around $15^{\circ} \mathrm{C}$ ) were operated successfully.

As an intermediate step between a single rod and the entire TOB, the Cosmic Rack (Fig. 10) was designed and built at CERN. It provides support for 20 rods arranged in two slightly overlapping columns, where one is tilted by $8^{\circ}$ with respect to the other: a geometry similar to a slice of the Outer Barrel. The mechanics allow easy connection of power, optical fibers and cooling, and can be complemented with scintillators to trigger traversing cosmic muons. This setup is devoted to the development of the data-acquisition system, slow control and monitoring system, tracking and alignment software, and to tests of new hardware. Up to now, the Cosmic Rack has been operated successfully in the two beam tests in May and October 2004. On both occasions, only one column was partially equipped, housing two double-sided and four single-sided rods. In May, data were taken at room temperature and the system was mostly used to test prototypes of the final Front End Driver (FED9U, see [5]) and final power supplies. The configuration of such a complex system with about 26000 read-out channels was investigated in detail and improvements have been applied for the second beam test. This test represented the first validation of the system at a level of complexity that is significant compared to

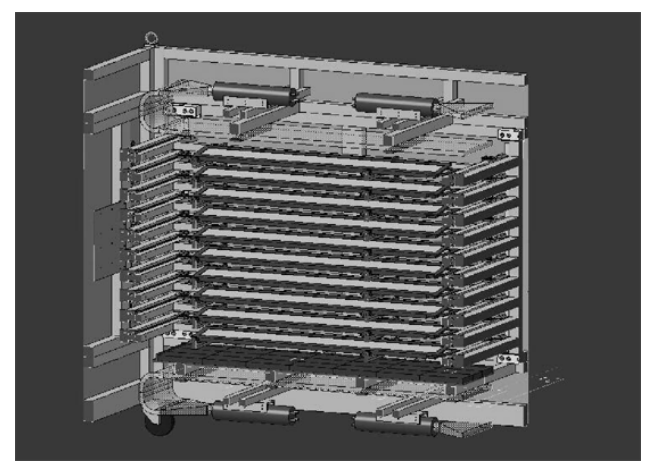

Fig. 10. The Cosmic Rack is an intermediate step between a single rod and the TOB. It allows system tests using up to 20 supermodules for the TOB.

the whole tracker, and allowed verifications of the noise performance ( $\sim 2000 e^{-}$in deconvolution mode) and the grounding scheme.

In the October beam test, the rods were tilted by $6^{\circ}$ with respect to the beam and an insulation allowed operation at low temperatures. This time, data acquisition software was used, which already makes use of a prototype of the final Run Control and Monitoring System (RCMS) [7].

The modules showed signal over noise values well above 20 in deconvolution mode and at $300 \mathrm{~V}$ bias voltage. A voltage scan showed that an over-depletion of about $100 \mathrm{~V}$ above the nominal full depletion voltage of $200 \mathrm{~V}$ was necessary to reach a plateau in the signal-to-noise ratio. It was also possible to reconstruct tracks with the standard CMS analysis software (ORCA) [8]. After an offline alignment of the layers, hit-to-track residuals show an rms of about $60 \mu \mathrm{m}$. An example is shown in Fig. 11 for layer 3 .

\section{PiXel Detector}

The order for the preproduction of final pixel sensors has been submitted and the delivery is expected by the end of 2004. Large effort was put on the improvement of radiation hardness. Finally, in spring 2004 it was decided to use $n^{+}$-strips with $p$-spray isolation on oxygenated $n$-type silicon (Fig. 12).

The pixel read-out chip was considerably modified by changing from DMILL to deep submicron technology, but the external operation stayed the same. The power consumption is now down to $29 \mu \mathrm{W}$ per pixel. In a 25 ns-beam test, it could be verified that the inefficiency is reduced to below $1 \%$ with the new chip (was about 5\% with DMILL).

\section{CONCLUSION}

The Strip Tracker Collaboration managed to recover from some technical difficulties in the production. Most of the assembly centres are now ready for an efficient production and the mechanical support structures have been delivered in time. The supermodules (rods, petals, half-cylinders) have been thoroughly tested both at the assembly centres and at beam tests with satisfying results.

The deep submicron version of the pixel read-out chip works well and a second iteration was submitted in August 2004. The preproduction of final barrel and end cap pixel sensors is ordered 


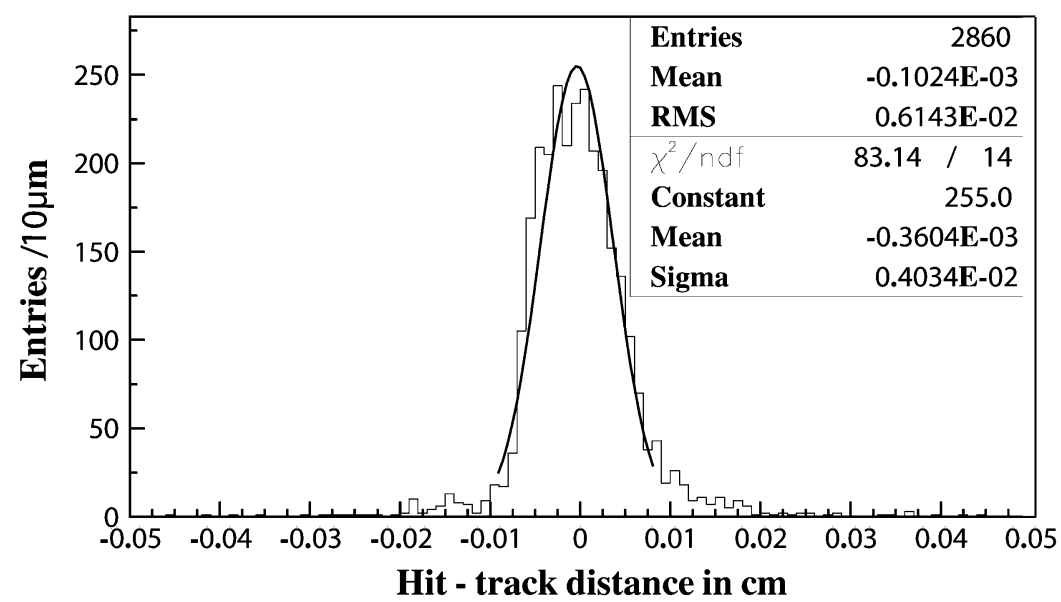

Fig. 11. Preliminary residuals of the rod in layer three using reconstructed tracks with hits in each layer [9]. The rods were inclined by $6^{\circ}$ with respect to the muon beam. The modules were operated in deconvolution mode at $-15^{\circ} \mathrm{C}$ and biased at $300 \mathrm{~V}$. Hit-to-track residuals show an rms of about $61 \mu \mathrm{m}$ (see statistical information in the plot). A Gaussian fit gives a core resolution of $40 \mu \mathrm{m}$ (see parameter "Sigma"). The investigated module has a pitch of $183 \mu \mathrm{m}$, and only tracks with hits in each layer are considered.

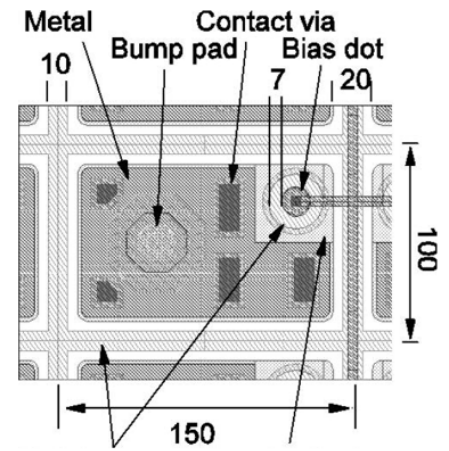

Full dose p-spray n-Implant

Fig. 12. New pixel design with $p$-spray isolation. The unit is $\mu \mathrm{m}$.

and the delivery is expected for the end of 2004. The main production of the pixel sensors is planned to start in summer 2005.

\section{REFERENCES}

[1] “Tracker Technical Design Report,", Geneva 23, Switzerland, 1998. The Tracker Project, CERN, CH-1211, CERN/LHCC 1998/006.

[2] “Addendum to the CMS Tracker TDR,", Geneva 23, Switzerland, 2000. The CMS Collaboration, CERN, CH-1211, CERN/LHCC 2000/016.

[3] M. Weber, Rod Cabling. Web link.

[4] M. Poettgens, "The CMS Silicon Tracker: Testing of Hybrids, Modules and Substructures at Operating Temperature," presented at the 2004 IEEE Nuclear Science Symposium, Rome, Italy, 2004.

[5] C. Foudas, "The cms tracker readout front end driver," presented at the 2004 IEEE Nuclear Science Symp., Rome, Italy, 2004.

[6] F. Drouhin, "The CERN CMS tracker control system," presented at the 2004 IEEE Nuclear Science Symp., Rome, Italy, 2004.

[7] "Data Acquisition and High-Level Trigger,", Geneva 23, Switzerland, vol. 2, Tech. Design Rep., 2002. The CMS TriDAS Project, CERN, CH-1211, CERN/LHCC 2002/026.

[8] S. Wynhoff, CMS OO Reconstruction. Web link.

[9] I. Reid, "Preliminary Results of Track Reconstruction Studies with ORCA," unpublished, 2004. 